OPEN ACCESS

Edited by: Huimao Zhang, First Affiliated Hospital of Jilin University, China

Reviewed by:

Subathra Adithan, Jawaharlal Institute of Postgraduate Medical Education and Research (JIPMER), India

Amrita Guha,

Tata Memorial Hospital, India

${ }^{*}$ Correspondence: Ming Zhang zhangming01@xjtu.edu.cn

Specialty section: This article was submitted to Cancer Imaging and Image-directed Interventions, a section of the journal

Frontiers in Oncology

Received: 29 January 2021 Accepted: 11 May 2021 Published: 04 June 2021

Citation:

Chen W, Zhang T, Xu L, Zhao L, Liu H, Gu LR, Wang $D Z$ and Zhang $M$ (2021) Radiomics Analysis of

Contrast-Enhanced CT for Hepatocellular Carcinoma Grading. Front. Oncol. 11:660509. doi: 10.3389/fonc.2021.660509

\section{Radiomics Analysis of Contrast- Enhanced CT for Hepatocellular Carcinoma Grading}

\author{
Wen Chen ${ }^{1,2}$, Tao Zhang ${ }^{2}$, Lin Xu ${ }^{2}$, Liang Zhao ${ }^{3}$, Huan Liu ${ }^{4}$, Liang Rui Gu ${ }^{5}$, \\ Dai Zhong Wang ${ }^{6}$ and Ming Zhang ${ }^{1 *}$
}

${ }^{1}$ Department of Medical Imaging, The First Affiliated Hospital of Xi'an Jiaotong University, Xi'an, China, ${ }^{2}$ Department of Radiology, Taihe Hospital, Hubei University of Medicine, Shiyan, China, ${ }^{3}$ Precision Medicine Research Center, Taihe Hospital, Hubei University of Medicine, Shiyan, China, ${ }^{4}$ GE Healthcare, Shanghai, China, ${ }^{5}$ Department of Radiology, Shanghai Sixth People's Hospital, Shanghai, China, ${ }^{6}$ Department of Pathology, Taihe Hospital, Hubei University of Medicine, Shiyan, China

Objectives: To investigate the value of contrast-enhanced computer tomography (CT)based on radiomics in discriminating high-grade and low-grade hepatocellular carcinoma (HCC) before surgery.

Methods: The retrospective study including 161 consecutive subjects with HCC which was approved by the institutional review board, and the patients were divided into a training group ( $n=112)$ and test group $(n=49)$ from January 2013 to January 2018. The least absolute shrinkage and selection operator (LASSO) was used to select the most valuable features to build a support vector machine (SVM) model. The performance of the predictive model was evaluated using the area under the curve (AUC), accuracy, sensitivity, and specificity.

Results: The SVM model showed an acceptable ability to differentiate high-grade from low-grade HCC, with an AUC of 0.904 in the training dataset and 0.937 in the test dataset, accuracy (92.2\% versus $95.7 \%)$, sensitivity(82.5\% versus $88.0 \%)$, and specificity $(92.7 \%$ versus 95.8\%), respectively.

Conclusion: The machine learning-based radiomics reflects a better evaluating performance in differentiating HCC between low-grade and high-grade, which may contribute to personalized treatment.

Keywords: radiomics, machine learning, support vector machine, hepatocellular carcinoma, grading

\section{INTRODUCTION}

Hepatocellular carcinoma (HCC) is the most common malignant tumor and predicted to the fourth leading cause of cancer death worldwide in 2018 (1-3). More than 300,000 people died in China because of liver cancer every year, accounting for $51 \%$ of liver cancer deaths worldwide (3). Surgical resection is the most effective treatment for patients with HCC. Patients who meet Milan criteria or undergo downstaging of their tumors to be within the Milan criteria are preferred for live transplantation $(4,5)$. The survival rates exceed $70 \%$ during 5 years, with recurrence in less than $15 \%$ of patients, who met Milan 
criteria and received a liver transplant (6). HCC is prone to metastasis and recurrence. However, the long-term prognosis of HCC patients is still unsatisfactory, although favorable results in terms of survival and recurrence have been reported based on highly selected patients $(7,8)$. The pathological grading of HCC plays an essential role in determining the patient's prognosis. The current study reflected that pathological grading is a risk factor of overall early recurrence in $\operatorname{HCC}(9,10)$. Intrahepatic recurrence and extrahepatic metastasis are more likely to occur in high-grade HCC tumors than low-grade tumors (11). Therefore, accurate preoperative prediction for HCC grading is crucial for treatment planning.

Medical imaging patterns of non-invasive contrast-enhanced CT in HCC patients are essential for accurate estimates of the clinical-stage, prognosis clinical decision-making, and determination of follow-up in primary hospitals. Contrastenhanced CT provides information about tumor vascularization. Studies have shown that there was a significant correlation between pathological grade and radiological enhancement on contrastenhanced CT (12), Only 46 patients were included in the study. Texture analysis based on CT images was reported to evaluate the differentiation and grade both of pancreatic carcinoma esophageal and renal cell carcinoma $(13,14)$.

Radiomics converts imaging data into a high-dimensional mineable feature space using many automatically extracted datacharacterization algorithms $(15,16)$. Quantitative features based on intensity, shape, and texture could be reflected in much information on tumor phenotype (17). Radiomics signatures have been proven to reflect tissue heterogeneity $(18,19)$. CT-based radiomics has been proven to discriminate tumor stage and grade in colorectal cancer $(20,21)$. Recent studies have shown that there is a correlation between medical imaging based on texture features and radiomics signatures and pathological grading (22-24).

However, there are few studies based on thin-portal phase CT radiomics to predict hepatocellular carcinoma grade. Therefore, we aimed to investigate the value of contrast-enhanced CT-based on portal venous radiomics signatures to distinguish HCC grade preoperatively.

\section{MATERIALS AND METHODS}

\section{Patients}

The retrospective study was approved by the institutional review board, and the informed consent requirement was waived. The inclusion criteria were as follows: (1) patients who underwent surgical resection and were pathologically confirmed as HCC with a usable histological report according to Edmondson-Steiner grades; (2) patients who underwent liver contrast-enhanced CT within two weeks before operation; (3) Without previous treatment with patients such as radiofrequency ablation, transcatheter arterial chemoembolization (TACE), liver resection or percutaneous ethanol injection; and (4) the quality of the images satisfied the needs of analysis and have completed the portal venous phase CT images and clinical and pathological data. The patients with HCC with a contrast-enhanced CT examination in our institute were recruited from January 2013 to January 2018. The enrolled 161 patients with HCC, were classified into the training dataset [112 patients; 86 males (76.8\%) and 26 women (23.2\%)], with a median age of 53 years (range 25 to 71 years)] and the test dataset [ 49 patients; 40 males $(81.7 \%)$ and nine females $(8.3 \%)$, with a median age of 57 years (range 28 to 74 years)]. Baseline clinicopathologic data, including gender, age, preoperative AFP level, were derived from the institution archives. Finally, a total of 161 subjects were selected from the total 456 patients in our research, and the details are shown in Figure 1.

\section{Assessment of Histologic Grade}

Histological grading data of HCC tumors were obtained from pathology reports reviewed by the pathologist. Histological grade was postoperatively determined as low- and high-grade. Edmondson grades I, I-II, and II correspond to Low-grade tumors, and Edmondson grades II-III, III, III-IV, and IV correspond to high-grade tumors (25). There was inconsistent differentiation in the tumor. Tumor cells of different pathological grades could be contained in the same mass. The larger one is determined as the pathological grade of the tumor (26).

\section{Image Acquisition}

All patients underwent 64 slices multidetector CT scanner of the liver (Optima CT660 or LightSpeed VCT, GE Healthcare), parameters were as follows: for non-enhanced studies and the hepatic arteriovenous phase, the gantry rotation time is $0.6 \mathrm{~s}$, and the equilibrium phase is $0.8 \mathrm{~s}$; the cross-sectional thickness is $5 \mathrm{~mm}$; the table speed is $27.5 \mathrm{~mm} / \mathrm{s} ; 120 \mathrm{kVp}$; and $160-440 \mathrm{~mA}$. Patients imaged with a CT scanner in a craniocaudal direction. The scan range is from the dome to the lower liver. Non-ionic contrast medium (Iohexol Injection) administered at a total dose of 70-80 $\mathrm{ml}$ based on body weight $(0.9 \mathrm{ml} / \mathrm{kg}), 2.5-3.0 \mathrm{ml} / \mathrm{s}$ through a 20 gauge venous cannula placed in the antecubital vein. For triphasic acquisitions, scanning started with a $30 \mathrm{~s}$ scan delay (about 30-35 s after injection of the contrast agent) for the hepatic arterial phase. Thirty-five seconds after the endpoint of the hepatic arterial phase (about 65-70 s after injection of the contrast agent), the scans for the portal venous phase were obtained. Delayed phase images reached $120 \mathrm{~s}$ (about 150-180 s after injection of the contrast agent) (27).

\section{Tumor Segmentation}

Tumor segmentation was performed on the portal venous phase CT images, retrieved from the picture archiving and communication system (PACS). The images were loaded into the ITK-SNAP software (open-source software http://www.itksnap.org) for manual segmentation, and a three-dimensional volume of interest (VOI) that covered the whole tumor was delineated in the images respectively segmented by a radiologist with over five years of experience in abdominal imaging. The procedure is shown in Figure 2.

\section{Radiomics Feature Extraction and Selection}

After integrating the VOI that covered the whole tumor images, a three-dimensional radiomics feature was extracted from the CT images with the Artificial Intelligence Kit software (AK, version 3.2.2, GE Healthcare). A total of 396 radiomics features from each patient were generated based on the following five categories: Histogram, shape, Gray-level co-occurrence matrix (GLCM), Gray-level size 


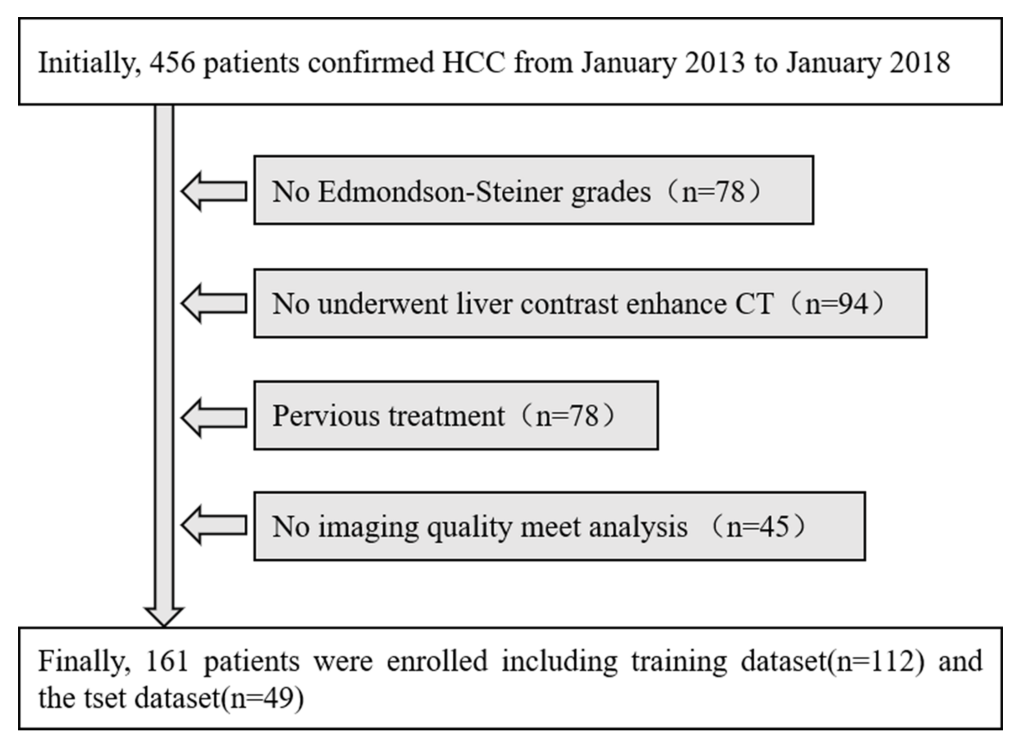

FIGURE 1 | Flowchart of the inclusion and exclusion processes.

zone matrix (GLSZM), and Run-length matrix (GLRLM). Most features defined comply with feature definitions as described by the Imaging Biomarker Standardization Initiative (IBSI).

Thirty CT images were randomly chosen for the second segmentation by two experienced radiologists (twice by reader one and once by reader 2 , with eight and thirteen years of clinical experience in the abdominal study). Intra- and interclass correlation coefficients (ICC) were applied to assess the stability and reproducibility to find out robust features. Based on the twice feature extraction by reader 1, the intra-observer ICCs were calculated. Meanwhile, the interobserver ICCs were obtained based on the first-extracted features by reader one and those by reader 2 . Generally, ICC $>0.75$ was considered to be excellent in reproducibility (28). The remaining tumor segmentation for feature extraction was performed by reader 1 .

All patients were randomly divided into two independent datasets with a ratio of 7:3 using stratified sampling. The feature scaling method was employed before dimensionality reduction to decrease the difference in radiomics features. First, The general univariate analysis was used to select features. The least absolute shrinkage and selection operator (LASSO) was applied to select the most useful features from the primary data in the training dataset. The Heatmap of the model in the training and test samples is shown in Figure 3. Detailed radiomics parameters and remained features are shown in the Supplementary Material.

\section{Establishment of the Model Based on Machine Learning}

The most predictive features were applied to establish an optimal SVM model using a grid search method with 5-fold crossvalidation. The AUC, sensitivity, specificity, positive predictive value (PPV), negative predictive value (NPV), and accuracy were further calculated in the dataset. And independently validated in the test dataset to evaluate prediction accuracy.

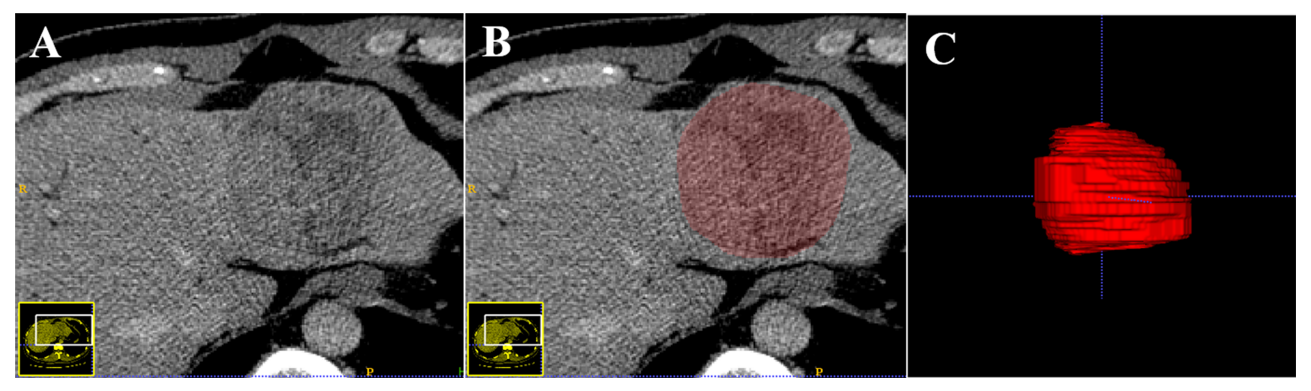

FIGURE 2 | An example of the manual segmentation in hepatocellular carcinoma. The portal venous phase computed tomography (CT) image (A). Manual segmentation on the same axial slice (B). Generation of a 3D ROI (C). 


\section{Statistical Analysis}

Continuous variables were analyzed with the Shapiro-Wilk test to determine their distributions. The Student's t-test and the MannWhitney $U$ test were used to determine whether the characteristic features were significantly different between the low- and high-grade HCC groups in the training dataset and test dataset. The statistical significance levels reported in this study were all two-sided, and a $P$ value $<.05$ was considered statistical significance. All statistical analyses were performed using IPMs 2.0 (IPM statistics, GE healthcare).

\section{RESULTS}

\section{Clinical Characteristics and Pathologic Findings}

The training dataset consisted of 86 males and 26 females. The mean age in the low grade of HCC in the training dataset is $56.45 \pm$ 10.44; range from 20-69 years. The mean age of high grade is $49.74 \pm$ 8.58 years; content from 25-78years, which has a significant difference $(p<0.01)$. The test dataset included 40 males and nine females (mean age, $51.38 \pm 8.22$; range, $24-83$ years in low grade; mean age, $51.88 \pm 10.74$; range, $25-72$ years in high grade). No statistically significant differences existed in gender between the training and the test datasets $(p=0.317 ; p=0.662)$. Clinical characteristics were detailedly shown in Table 1. No significant difference was found in the AFP level between patients with lowgrade and high-grade HCC either in the training dataset or test dataset $(p=0.186 ; p=0.150)$. Among 161 patients underwent surgical resection, including laparoscopy or laparotomy. Of these, 79 were low-grade hepatocellular carcinoma, and 82 were high-grade hepatocellular carcinoma.

\section{Reproducibility of Radiomics Feature Extraction}

A total of 396 radiomics features were extracted for each patient. Among these radiomics features, 312 features were considered excellent reproducibility with ICC $>0.75$ in intra-and interobserver. Therefore, the 312 robust features of each patient were used for further selection. Finally, seven features with non-zero coefficients were eventually remained from the 312 radiomics features using LASSO logistic regression (Figure 4).

\section{Performance of SVM}

The model based on SVM on the portal venous phase CT images performed well on high-grade patients from low-grade patients. With an AUC of 0.904 in the training dataset, the test dataset with an AUC of 0.937 (Figure 5). The other predictive parameters (sensitivity, specificity, PPV, NPV, and accuracy) of SVM are shown in Table 2.
A

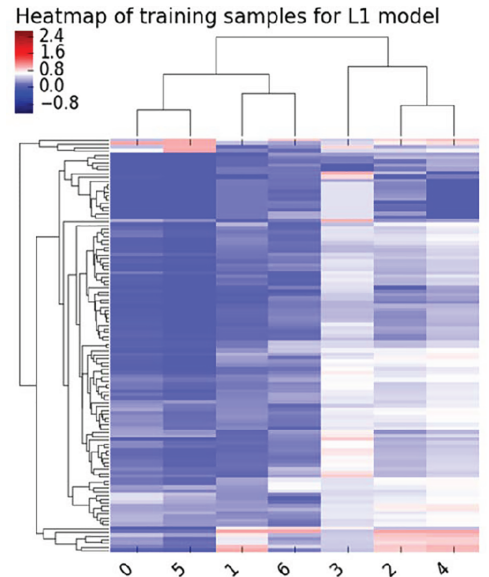

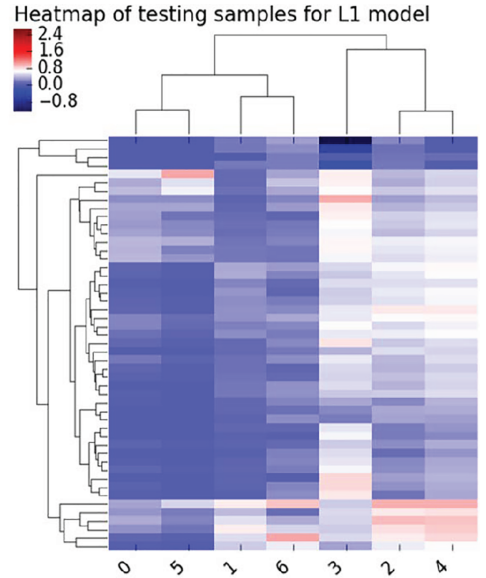

FIGURE 3 | Heatmap of the model in the training (A) and test samples (B) for L1 model.

TABLE 1 | Baseline characteristics of patients in training dataset and test dataset.

\begin{tabular}{|c|c|c|c|c|c|c|}
\hline Characteristics & \multicolumn{3}{|c|}{ Training dataset } & \multicolumn{3}{|c|}{ Test dataset } \\
\hline $\begin{array}{l}\text { Age, mean } \pm S D, y \\
\text { Gender }<N\end{array}$ & $56.45 \pm 10.44$ & $49.74 \pm 8.58$ & $<0.01$ & $51.38 \pm 8.22$ & $51.88 \pm 10.74$ & 0.855 \\
\hline Male & 40 & 46 & 0.317 & 19 & 21 & 0.662 \\
\hline Female & 15 & 11 & & 5 & 4 & \\
\hline $\begin{array}{l}\text { AFP (ug/L) Median } \\
\text { (IQR) }\end{array}$ & $\begin{array}{c}20.5 \\
(8.21,42.3)\end{array}$ & $\begin{array}{c}32.4 \\
(7.82,45.95)\end{array}$ & 0.186 & $\begin{array}{c}20.55 \\
(6.95,35.54)\end{array}$ & $\begin{array}{c}27.3 \\
(10.54,61.82)\end{array}$ & 0.15 \\
\hline
\end{tabular}




\section{DISCUSSION}

Hepatocellular carcinoma (HCC) is the most common tumor of liver cancers accounting for more than $90 \%$ (6). Most of the world's HCC cases are found in the Asia-Pacific region, where annual HCCrelated mortality rates have risen significantly over the last 20 years (29). HCC has become a major emerging public health problem in the Asia-Pacific region. Radiomics has been proven useful in tumor grade in clear cell renal cell carcinoma, soft tissue sarcomas and Colorectal Adenocarcinoma (21, 30, 31). The SVM classifier, a specific type of supervised machine-learning method, has been used to predict the grade of the glioma (32-34) and clear cell renal cell (35). As the results showed, The SVM model was finally developed after the LASSO regression analysis to discriminate the grade of HCC in both the training dataset $(p<0.05)$ and the test dataset $(p$ $<0.05$ ). It indicates that radiomics features on the portal venous phase CT images can be used to detect tiny differences in the density of tumors.

The AUC on the portal phase CT-based SVM for preoperative prediction of the grade of hepatocellular carcinoma is 0.904 and
A

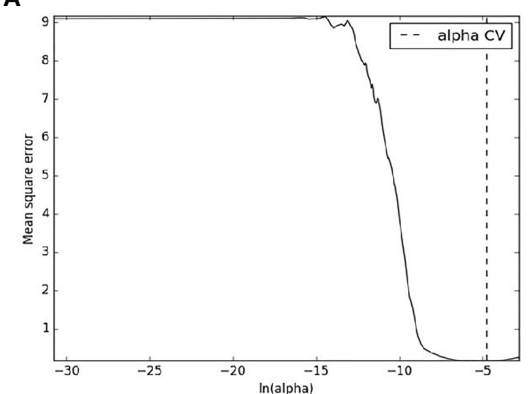

B

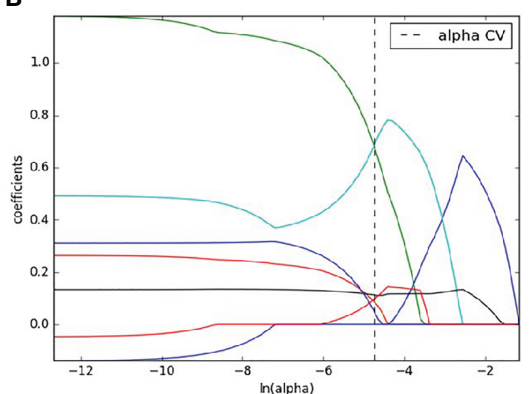

FIGURE 4 | Radiomics features selection with LASSO binary logistic regression method. The mean square error was plotted versus the In (alpha) sequence (A); The coefficient profile plot was plotted versus the In (alpha) sequence (B).
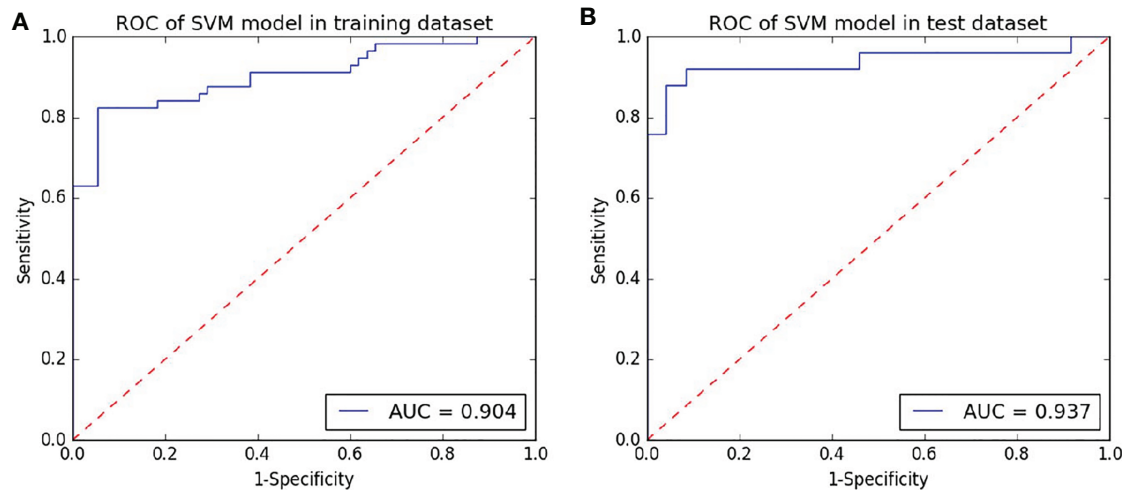

FIGURE 5 | Receiver operating characteristic curves (ROC) of the portal phase CT-based SVM for preoperative prediction of the grade of hepatocellular carcinoma in the training and testing datasets. (A) the ROC curve of the radiomics signature based on the portal phase CT based on the training dataset. (B) the ROC curve of radiomics signature based on the portal phase CT for the test dataset.

TABLE 2 | The predictive performance of the SVM model for preoperative the grade of hepatocellular carcinoma based on contrast-enhanced CT.

\begin{tabular}{lccccc}
\hline Predictive Performance & AUC & SEN & SPE & ACC & PPV \\
\hline Training dataset & 0.904 & 0.825 & 0.927 & 0.922 & 0.922 \\
Test dataset & 0.937 & 0.880 & 0.958 & 0.957 & 0.957
\end{tabular}


0.937 in the training and test datasets. A published study showed that the radiomics signature based on T1WI or T2WI images showed performance in predicting the HCC grade (with AUC of 0.712 and 0.722 in the test dataset) (22). Mao et al. showed that the radiomics signatures based on arterial phase Contrast-enhanced computed tomography images could successfully distinguish pathological grades of HCC, with the AUC of 0.731 and 0.718 in the training and test datasets (24), which are lower than the AUC of the SVM model developed in our study either in training dataset or test dataset. This result may be related to section thickness and selection of the various phases of contrast enhancement. The section thickness chose is 0.625 or $1.25 \mathrm{~mm}$ and we chose the portal venous phase in our study. Approximately $80-90 \%$ of HCCs are hypervascular lesions, arterial phase of imaging could increase enhancement in the tumor parenchyma. Due to differences in tumor biology, measurements of HCC will vary at the various phases of contrast enhancement (36). Mapping the size of hepatocellular carcinoma (HCC) on images plays an important role in accurately capturing a three-dimensional region of interest. Research has suggested that the portal venous phase may be optimal for measuring HCC on MRI (37), which is similar to CT. They hypothesized that benign reactivity or perfusion-related changes may occur in the liver parenchyma around the tumor during the arterial phase and may present as transient congestion during this phase, leading to measurement bias. The tumor will be with wash out on the portal venous phase, making it easier to show the boundary of the tumor.

Studies have shown that the texture features based on arterial phase CT images are associated with pathological grades of HCC (23). Texture feature based on Gd-DTPA-enhanced MR images showed better diagnostic efficacy (with AUCs of 0.827-0.918) (38) because MR images providing more information about tumor heterogeneity. However, the case number was limited, It may lead to a possible risk of data overfitting.

Some studies indicate that the model which combined clinical factors with the radiomic model outperformed compared with other models $(22,24)$. Research shows AFP level was an independent factor that could discriminate between high-grade and low-grade HCC (22). However, there is no significant difference between high-grade and low-grade HCC in both the training and test datasets $(p=0.186$ and $p=0.15$ ). It may be due to the high AFP level in some patients in this study, resulting in the imbalance of the AFP level.

There are several limitations to our research. First, the number of HCC was limited and it was a single-center retrospective study. Therefore, more cases are needed for future studies and further multicenter cohorts should be conducted. Second, the portal venous phase image was merely considered, which might somehow miss some useful information for the hepatic arterial

\section{REFERENCES}

1. Ferlay J, Soerjomataram I, Dikshit R, Eser S, Mathers C, Rebelo M, et al. Cancer Incidence and Mortality Worldwide: Sources, Methods and Major Patterns in GLOBOCAN 2012. Int J Cancer (2015) 136(5):E359-86. doi: 10.1002/ijc. 29210

2. Njei B, Rotman Y, Ditah I, Lim JK. Emerging Trends in Hepatocellular Carcinoma Incidence and Mortality. Hepatology (2015) 61(1):191-9. doi: 10.1002/hep.27388 and hepatic venous phases. Thus, the phase images should also be incorporated in future studies. Third, the etiology of liver cancer hasn't been classified. thus, further research is needed to determine whether our findings would be influenced by different etiology such as hepatitis B, hepatitis C-related liver diseases, and alcoholrelated cirrhosis.

\section{CONCLUSIONS}

An SVM model by radiomics signature based on contrastenhanced CT images may be useful as a new approach to predicting the histological grade of HCC before the operation.

\section{DATA AVAILABILITY STATEMENT}

The original contributions presented in the study are included in the article/Supplementary Material. Further inquiries can be directed to the corresponding author.

\section{ETHICS STATEMENT}

The studies involving human participants were reviewed and approved by Letter of Medical Ethics Committee of Shiyan Taihe Hospital. The patients/participants provided their written informed consent to participate in this study.

\section{AUTHOR CONTRIBUTIONS}

WC, TZ, and MZ reviewed and drafted the manuscript. TZ, RG, and DW did the literature search. LX and LZ modified the figures. HL and WC have revised and edited the manuscript. All authors contributed to the article and approved the submitted version.

\section{FUNDING}

This study was funded by the research grant from the Health Commission of Hubei Province scientific research project (WJ2021M047).

\section{SUPPLEMENTARY MATERIAL}

The Supplementary Material for this article can be found online at: https://www.frontiersin.org/articles/10.3389/fonc.2021. 660509/full\#supplementary-material

3. Bray F, Ferlay J, Soerjomataram I, Siegel RL, Torre LA, Jemal A. Global Cancer Statistics 2018: GLOBOCAN Estimates of Incidence and Mortality Worldwide for 36 Cancers in 185 Countries. CA Cancer J Clin (2018) 68 (6):394-424. doi: 10.3322/caac.21492

4. Nitta H, Allard MA, Sebagh M, Karam V, Ciacio O, Pittau G, et al. Predictive Model for Microvascular Invasion of Hepatocellular Carcinoma Among Candidates for Either Hepatic Resection or Liver Transplantation. Surgery (2019) 165(6):1168-75. doi: 10.1016/j.surg.2019.01.012 
5. Pinna AD, Yang T, Mazzaferro V, De Carlis L, Zhou J, Roayaie S, et al. Liver Transplantation and Hepatic Resection can Achieve Cure for Hepatocellular Carcinoma: Erratum. Ann Surg (2019) 268:868-75. doi: 10.1097/ SLA.0000000000002889

6. Kulik L, El-Serag HB. Epidemiology and Management of Hepatocellular Carcinoma. Gastroenterology (2019) 156(2):477-491.e1. doi: 10.1053/j.gastro. 2018.08.065

7. Tabrizian P, Jibara G, Shrager B, Schwartz M, Roayaie S. Recurrence of Hepatocellular Cancer After Resection: Patterns, Treatments, and Prognosis. Ann Surg (2015) 261(5):947-55. doi: 10.1097/SLA.0000000000000710

8. Huang PY, Wang CC, Lin CC, Lu SN, Wang JH, Hung CH, et al. Predictive Effects of Inflammatory Scores in Patients With BCLC 0-a Hepatocellular Carcinoma After Hepatectomy. J Clin Med (2019) 8(10). doi: 10.3390/jcm8101676

9. Zhou L, Rui JA, Wang SB, Chen SG, Qu Q. Clinicopathological Predictors of Poor Survival and Recurrence After Curative Resection in Hepatocellular Carcinoma Without Portal Vein Tumor Thrombosis. Pathol Oncol Res (2015) 21(1):131-8. doi: 10.1007/s12253-014-9798-2

10. Zhou L, Rui JA, Zhou WX, Wang SB, Chen SG, Qu Q. Edmondson-Steiner Grade: A Crucial Predictor of Recurrence and Survival in Hepatocellular Carcinoma Without Microvascular Invasio. Pathol Res Pract (2017) 213 (7):824-30. doi: 10.1016/j.prp.2017.03.002

11. Pérez-Saborido B, de los Galanes SJ, Menéu-Díaz JC, Romero CJ, Elola-Olaso AM, Suárez YF, et al. Tumor Recurrence After Liver Transplantation for Hepatocellular Carcinoma: Recurrence Pathway and Prognostic Factors. Transplant Proc (2007) 39(7):2304-7. doi: 10.1016/j.transproceed.2007.06.059

12. Tarhan NC, Hatipoğlu T, Ercan E, Bener M, Keleş G, Başaran C, et al. Correlation of Dynamic Multidetector CT Findings With Pathological Grades of Hepatocellular Carcinoma. Diagn Interv Radiol (2011) 17(4):328-33. doi: 10.4261/1305-3825.DIR.2682-09.3

13. Longlong Z, Xinxiang L, Yaqiong G, Wei W. Predictive Value of the Texture Analysis of Enhanced Computed Tomographic Images for Preoperative Pancreatic Carcinoma Differentiation. Front Bioeng Biotechnol (2020) 8:719. doi: 10.3389/fbioe.2020.00719

14. Yaşar S, Voyvoda N, Voyvoda B, Özer T. Using Texture Analysis as a Predictive Factor of Subtype, Grade and Stage of Renal Cell Carcinoma. Abdom Radiol (NY) (2020) 45(11):3821-30. doi: 10.1007/s00261-020-02495-6

15. Lambin P, Rios-Velazquez E, Leijenaar R, Carvalho S, van Stiphout RG, Granton P, et al. Radiomics: Extracting More Information From Medical Images Using Advanced Feature Analysis. Eur J Cancer (2012) 48(4):441-6. doi: 10.1016/j.ejca.2011.11.036

16. Kumar V, Gu Y, Basu S, Berglund A, Eschrich SA, Schabath MB, et al. Radiomics: The Process and the Challenges. Magn Reson Imaging (2012) 30 (9):1234-48. doi: 10.1016/j.mri.2012.06.010

17. Gillies RJ, Kinahan PE, Hricak H. Radiomics: Images are More Than Pictures, They Are Data. Radiology (2016) 278(2):563-77. doi: 10.1148/radiol.2015151169

18. Yip C, Landau D, Kozarski R, Ganeshan B, Thomas R, Michaelidou A, et al. Primary Esophageal Cancer: Heterogeneity as Potential Prognostic Biomarker in Patients Treated With Definitive Chemotherapy and Radiation Therapy. Radiology (2014) 270(1):141-8. doi: 10.1148/radiol.13122869

19. Davnall F, Yip CS, Ljungqvist G, Selmi M, Ng F, Sanghera B, et al. Assessment of Tumor Heterogeneity: An Emerging Imaging Tool for Clinical Practice. Insights Imaging (2012) 3(6):573-89. doi: 10.1007/s13244-012-0196-6

20. Liang C, Huang Y, He L, Chen X, Ma Z, Dong D, et al. The Development and Validation of a CT-Based Radiomics Signature for the Preoperative Discrimination of Stage I-II and Stage III-IV Colorectal Cancer. Oncotarget (2016) 7(21):31401-12. doi: 10.18632/oncotarget.8919

21. Huang X, Cheng Z, Huang Y, Liang C, He L, Ma Z, et al. CT-Based Radiomics Signature to Discriminate High-Grade From Low-Grade Colorectal Adenocarcinoma. Acad Radiol (2018) 25(10):1285-97. doi: 10.1016/j.acra. 2018.01.020

22. Wu M, Tan H, Gao F, Hai J, Ning P, Chen J, et al. Predicting the Grade of Hepatocellular Carcinoma Based on Non-Contrast-Enhanced MRI Radiomics Signature. Eur Radiol (2019) 29(6):2802-11. doi: 10.1007/s00330-018-5787-2

23. Oh J, Lee JM, Park J, Joo I, Yoon JH, Lee DH, et al. Hepatocellular Carcinoma: Texture Analysis of Preoperative Computed Tomography Images Can Provide Markers of Tumor Grade and Disease-Free Survival. Korean J Radiol (2019) 20(4):569-79. doi: 10.3348/kjr.2018.0501
24. Mao B, Zhang L, Ning P, Ding F, Wu F, Lu G, et al. Preoperative Prediction for Pathological Grade of Hepatocellular Carcinoma Via Machine LearningBased Radiomics. Eur Radiol (2020) 30(12):6924-32. doi: 10.1007/s00330020-07056-5

25. Edmondson HA, Steiner PE. Primary Carcinoma of the Liver: A Study of 100 Cases Among 48,900 Necropsies. Cancer (1954) 7(3):462-503. doi: 10.1002/ 1097-0142(195405)7:3<462::AID-CNCR2820070308>3.0.CO;2-E

26. Huang Y, Liu Z, He L, Chen X, Pan D, Ma Z, et al. Radiomics Signature: A Potential Biomarker for the Prediction of Disease-Free Survival in Early-Stage (I or II) non-Small Cell Lung Cancer. Radiology (2016) 281(3):947-57. doi: 10.1148/ radiol.2016152234

27. Zhang T, Pandey G, Xu L, Chen W, Gu L, Wu Y, et al. The Value of TTPVI in Prediction of Microvascular Invasion in Hepatocellular Carcinoma. Cancer Manag Res (2020) 12:4097-105. doi: 10.2147/CMAR.S245475

28. Gstoettner M, Sekyra K, Walochnik N, Winter P, Wachter R, Bach CM. Interand Intraobserver Reliability Assessment of the Cobb Angle: Manual Versus Digital Measurement Tools. Eur Spine J (2007) 16(10):1587-92. doi: 10.1007/ s00586-007-0401-3

29. Lozano R, Naghavi M, Foreman K, Lim S, Shibuya K, Aboyans V, et al. Global and Regional Mortality From 235 Causes of Death for 20 Age Groups in 1990 and 2010: A Systematic Analysis for the Global Burden of Disease Study 2010. Lancet (2012) 380(9859):2095-128. doi: 10.1016/S0140-6736(12)61728-0

30. Ding J, Xing Z, Jiang Z, Chen J, Pan L, Qiu J, et al. CT-Based Radiomic Model Predicts High Grade of Clear Cell Renal Cell Carcinoma. Eur J Radiol (2018) 103:51-6. doi: 10.1016/j.ejrad.2018.04.013

31. Wang H, Chen H, Duan S, Hao D, Liu J. Radiomics and Machine Learning With Multiparametric Preoperative Mri May Accurately Predict the Histopathological Grades of Soft Tissue Sarcomas. J Magn Reson Imaging (2020) 51(3):791-7. doi: 10.1002/jmri.26901

32. Qi C, Li Y, Fan X, Jiang Y, Wang R, Yang S, et al. A Quantitative SVM Approach Potentially Improves the Accuracy of Magnetic Resonance Spectroscopy in the Preoperative Evaluation of the Grades of Diffuse Gliomas. NeuroImage Clin (2019) 23:101835. doi: 10.1016/j.nicl.2019.101835

33. Tian Q, Yan LF, Zhang X, Zhang X, Hu YC, Han Y, et al. Radiomics Strategy for Glioma Grading Using Texture Features From Multiparametric MRI. J Magn Reson Imaging (2018) 48:1518-28. doi: 10.1002/jmri.26010

34. Vamvakas A, Williams SC, Theodorou K, Kapsalaki E, Fountas K, Kappas C, et al. Imaging Biomarker Analysis of Advanced Multiparametric MRI for Glioma Grading. Phys Med (2019) 60:188-98. doi: 10.1016/j.ejmp.2019.03.014

35. Nazari M, Shiri I, Hajianfar G, Oveisi N, Abdollahi H, Deevband MR, et al. Noninvasive Fuhrman Grading of Clear Cell Renal Cell Carcinoma Using Computed Tomography Radiomic Features and Machine Learning. Radiol Med (2020) 125:754-62. doi: 10.1007/s11547-020-01169-z

36. Hanna RF, Aguirre DA, Kased N, Emery SC, Peterson MR, Sirlin CB. CirrhosisAssociated Hepatocellular Nodules: Correlation of Histopathologic and MR Imaging Features. Radiographics (2008) 28:747-69. doi: 10.1148/rg.283055108

37. Seuss CR, Kim MJ, Triolo MJ, Hajdu CH, Rosenkrantz AB. Comparison of MRI Pulse Sequences for Prediction of Size of Hepatocellular Carcinoma At Explant Evaluation. AJR Am J Roentgenol (2014) 203:300-5. doi: 10.2214/AJR.13.11688

38. Zhou W, Zhang L, Wang K, Chen S, Wang G, Liu Z, et al. Malignancy Characterization of Hepatocellular Carcinomas Based on Texture Analysis of Contrast-Enhanced MR Images. J Magn Reson Imaging (2017) 45(5):1476-84. doi: $10.1002 /$ jmri.25454

Conflict of Interest: Author HL was employed by company GE Healthcare.

The remaining authors declare that the research was conducted in the absence of any commercial or financial relationships that could be construed as a potential conflict of interest.

Copyright (0) 2021 Chen, Zhang, Xu, Zhao, Liu, Gu, Wang and Zhang. This is an open-access article distributed under the terms of the Creative Commons Attribution License (CC BY). The use, distribution or reproduction in other forums is permitted, provided the original author(s) and the copyright owner(s) are credited and that the original publication in this journal is cited, in accordance with accepted academic practice. No use, distribution or reproduction is permitted which does not comply with these terms. 